\title{
Forecasting Foreign Currency Exchange Price using Long Short-Term Memory with K-Nearest Neighbor Method
}

\author{
Rudra Kalyan Nayak, S.Y.H. Pavitra, Ramamani Tripathy, K. Prathyusha
}

\begin{abstract}
With the growing population in the world, economic stability varies day by day. In case of India all banking transaction rules and regulations are taken by Reserve bank of India (RBI) whereas for other countries it is different. Therefore numerous academicians have projected their research on forecasting the currency exchange rate for diverse countryside. Foreign currency exchange rate prediction is a very pivotal task for international market. Hence researchers have explored different methods for predicting foreign currency exchange rate. In this work, we have taken Indian rupees (INR) with two different country's data set such as Japanese yen (JPY) andChinese Yuan (CNY)for daily, weekly and monthlyprediction beforehand. We implemented a hybrid model oflong short-term memory (LSTM) with K-nearest neighbour (KNN) which gives better opening price prediction accuracy on our dataset. The accuracy of the prediction results are measured by the help of performance standards such as mean absolute percentage error (MAPE) and root mean square error (RMSE).
\end{abstract}

Keywords: Currency exchange rate, LSTM, KNN, RBI.

\section{INTRODUCTION}

Forecasting foreign exchange rate is a very pivotal factor for international transaction environment because all country's economic condition has been determined by depending on currency exchange rate. It is not mandatory that today's currency exchange rate will be same in tomorrow market, so all the times exchange rate is fluctuating. Therefore people have their eye on currency exchange rate while transferring or receiving money internationally. There are so many factors available which might be responsible for fluctuation in exchange rates such as inflation rates, interest rates, country's current account / balance of payments, Government debt, terms of trade, political stability \& performance, recession, speculation etc. For this purpose economists

Revised Manuscript Received on December 30, 2019.

* Correspondence Author

Dr.Rudra Kalyan Nayak*, Assoc. Professor of CSE,KoneruLakshmaiah Education Foundation (Deemed to be University), AP, India.Email: rudrakalyannayak@gmail.com

S.Y.H. Pavitra, Student of 4th YearB.Tech (CSE), KoneruLakshmaiah Education Foundation (Deemed to be University), AP, India. Email:surepavitra@gmail.com

Dr.Ramamani Tripathy, Asst. Professor of MCA, United School of Business Management,Odisha, India. Email: ramatripathy1978@gmail.com

K. Prathyusha, Student of 4th YearB.Tech (CSE), KoneruLakshmaiah Education Foundation (Deemed to be University), AP, India. Email:mail2kprathyusha@gmail.com

(C) The Authors. Published by Blue Eyes Intelligence Engineering and Sciences Publication (BEIESP). This is an open access article under the CC BY-NC-ND license (http://creativecommons.org/licenses/by-nc-nd/4.0/) and shareholders constantly have a tendency to predict the future exchange rates so that they can have faith on forecasting to derive monetary value. According to current international economy scenario, accurately predicting the foreign exchange rate is of vital part for any future investment. Therefore different methods have been implemented by different researchers for forecasting the currency exchange rates [1-5].

However, as is the case with forecasts, approximately all of these techniques are full of complications and not any one of them can be said to be hundred percent effectual in obtaining the precise future currency exchange rate. So every time model has been changed with the dataset and each have their own restrictions. Upon that constraint the method may work. In the long-ago, numerous models have been projected for time series forecasting. Generally fashionable ones are recurrent neural networks (RNNs), Fuzzy Logic,auto regressive moving average (ARMA),auto regressive integrated moving average (ARIMA),support vector machine (SVM),convolutional neural network (CNN), etc. [6-11].

The main intention of this work is forecasting of future currency exchange rate for INR with JPY and CNY data using a novel hybrid forecasting model LSTM with KNN which would be helpful for all economists and shareholders in the world. From this analysis we have found that our proposed forecasting model achievedvery well results concerning MAPE and RMSE. Whole part of our manuscript is organised as follows: literature works are depicted in segment 2, segment 3 describes the dataset description with proposed model, next in segment 4 we provide the details about methodology and experimental results and in last segment we have discussed the conclusion part.

\section{LITERATURE WORKS}

So far as currency exchange price is concerned numerous methods have been adopted. Here proposed LSTM-K-NN model has been thought of considering the following literatures. The author Yao, Jingtaoet al. [12] have implemented neural network model for forecasting currency exchange rate. In this paper researcher emphasises on time series data and technical indicators for getting better currency exchange rate. Here they have taken the data set such as Japanese Yen, Deutsch Mark, British Pound, Swiss Franc and Australian Dollar etc. 
Again other scientistsPanda, Chakradhara et al. [13] have proposed a novel artificial neural network modelfor forecasting foreign exchange rate using weekly Indian rupee/US dollar data set.

In this paper author has compared their proposed model with other well-known techniques such as linear autoregressive and random walk models to aware about the accuracy among all.

Researcher concludes that their model was suitable for policy makers and investor's in the foreign exchange market.Authors $\mathrm{Yu}$, Lean et al. [14] have exposed theirexperiment on multistage nonlinear radial basis function (RBF) neural network ensemble learning for predicting the currency exchange rates. In this analysis they categorised their work in to number of stages. In first phase they have constructed single RBF neural network model. In next phase a conditional generalized variance (CGV) minimization method was used to choose the appropriate ensemble members. Finally in last phase, another RBF network was used for neural network ensemble for prediction purpose. For measuring the performance of the proposed model they have compared with other method and disclosed that forecasting based on proposed model gives better than those achieved using the other methods.Anastasakiset al. [15] have studied their research on forecasting foreign currency exchange rate using both parametric and non-parametric self-organising modelling algorithm for daily basis. The combined methodology is performing well using the dataset. The researchers Gill, S. S. et al. [16] have implemented their model neural networks for predicting currency exchange rate for Indian scenario. According to author forecasting currency exchange rate is a very tedious task for all scientist. Many more time series data with methods have been applied but all have not given that much of prediction accuracy. Therefore they have taken neural network model for forecasting currency exchange rate and it outperformedbetter using larger data set in lesser time.

\section{DISCUSSION ABOUT DATA SET AND PROPOSED MODEL}

\section{A. Dataset Description}

In every types of analysis, data set plays an important role. Without proper knowledge and accuracy of data investigation is useless. So, primary objective of every researcher is to concentrate on dataset before experiment. In this paper secondary data is usedand is divided in to 2 parts. One branch is taken for training and other part is chosen for testing. Here we took two different countries currency exchange data set such asJPY and CNY for monthly, weekly and daily basis from [17] 'http://in.investing.com/currencies'. The data was taken in the range from $3^{\text {rd }}$ August 2009 to $1^{\text {st }}$ August 2019. Out of 2780 samples 1946 samples are taken for training and 834 for testing.Sample data of JPY/INRis shown below in table-I [17](normalized dataset).
Table-I: Sample dataset of JPY/INR currency on daily

\begin{tabular}{|c|c|c|c|c|c|c|}
\hline 1 & Date & Price & Open & High & Low & Change \% \\
\hline 2 & Aug 01, 2019 & 0.6425 & 0.6329 & 0.6436 & 0.6301 & $1.51 \%$ \\
\hline 3 & Jul 31, 2019 & 0.6329 & 0.6342 & 0.6352 & 0.6322 & $-0.20 \%$ \\
\hline 4 & Jul 30, 2019 & 0.6342 & 0.6316 & 0.6348 & 0.6311 & $0.40 \%$ \\
\hline 5 & Jul 29, 2019 & 0.6316 & 0.6337 & 0.6343 & 0.6313 & $-0.33 \%$ \\
\hline 6 & Jul 28, 2019 & 0.6337 & 0.6337 & 0.6337 & 0.6337 & $0.00 \%$ \\
\hline 7 & Jul 26, 2019 & 0.6337 & 0.6364 & 0.6367 & 0.6331 & $-0.42 \%$ \\
\hline 8 & Jul 25, 2019 & 0.6364 & 0.6376 & 0.6395 & 0.6348 & $-0.19 \%$ \\
\hline 9 & Jul 24, 2019 & 0.6376 & 0.6378 & 0.6392 & 0.6372 & $-0.02 \%$ \\
\hline 10 & Jul 23, 2019 & 0.6378 & 0.6388 & 0.6391 & 0.637 & $-0.16 \%$ \\
\hline 11 & Jul 22, 2019 & 0.6388 & 0.6392 & 0.64 & 0.6383 & $-0.07 \%$ \\
\hline 12 & Jul 21, 2019 & 0.6392 & 0.6392 & 0.6392 & 0.6392 & $0.02 \%$ \\
\hline 13 & Jul 19, 2019 & 0.6391 & 0.6411 & 0.6415 & 0.6374 & $-0.30 \%$ \\
\hline 14 & Jul 18, 2019 & 0.6411 & 0.6378 & 0.6422 & 0.6376 & $0.52 \%$ \\
\hline 15 & Jul 17, 2019 & 0.6378 & 0.6348 & 0.6382 & 0.6341 & $0.47 \%$ \\
\hline 16 & Jul 16, 2019 & 0.6348 & 0.6352 & 0.637 & 0.6337 & $-0.06 \%$ \\
\hline 17 & Jul 15, 2019 & 0.6351 & 0.6354 & 0.6357 & 0.6344 & $-0.04 \%$ \\
\hline 18 & Jul 14, 2019 & 0.6354 & 0.6354 & 0.6354 & 0.6354 & $0.00 \%$ \\
\hline 19 & Jul 12, 2019 & 0.6354 & 0.6303 & 0.6362 & 0.6302 & $0.81 \%$ \\
\hline 20 & Jul 11, 2019 & 0.6303 & 0.6315 & 6337 & 0.6303 & $-0.19 \%$ \\
\hline 21 & Jul 10, 2019 & 0.6315 & 0.6294 & 0.6317 & 0.6285 & $0.33 \%$ \\
\hline 22 & Jul 09, 2019 & 0.6294 & 0.6307 & 0.6331 & 0.6294 & $-0.21 \%$ \\
\hline 23 & Jul 08, 2019 & 0.6307 & 0.6311 & 0.6346 & 0.6302 & $-0.06 \%$ \\
\hline 24 & Jul 07, 2019 & 0.6311 & 0.6311 & 0.6311 & 0.6311 & $-0.01 \%$ \\
\hline 25 & Jul 05, 2019 & 0.6312 & 0.6356 & 0.6374 & 0.6304 & $-0.70 \%$ \\
\hline
\end{tabular}

\section{B. Proposed Model}

Global market fluctuates due to various factors. According to current international economy scenario, accurately predicting the foreign exchange rate is of vital part for any future investment. Therefore different methods have been implemented by different researchers for forecasting the currency exchange rates. In this manuscript we have adopted novel hybrid model for forecasting the currency exchange rate.

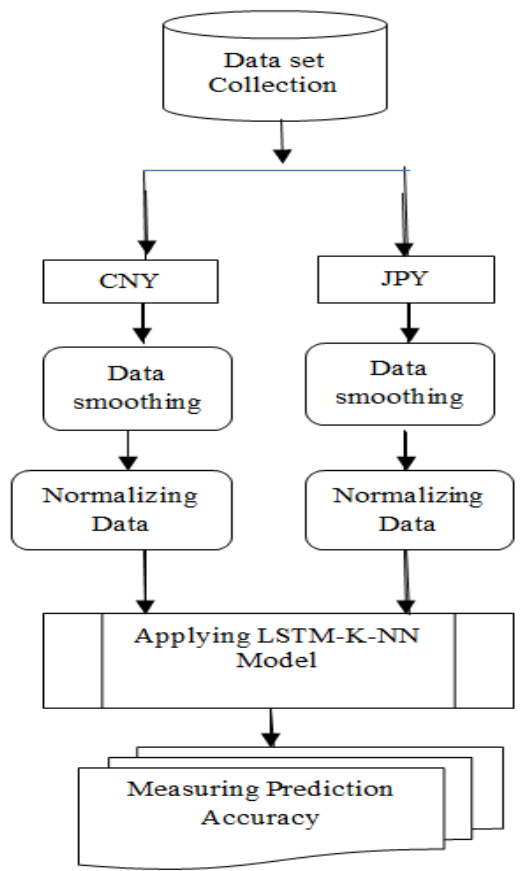

Figure 1: Flow chart of proposed model

Figure 1 represents the flow of proposed model procedure. In our research we have taken currency exchange dataset of two countries like JPY and CNY for prediction reason.

In stage 1 we have retrieved currency exchange data according to daily, weekly and monthly for aforesaid two countries. Then we have smoothed our retrieved data using technical indicators. 
The exact aim of smoothing is to exterminate noise and well again expose the signal of the underlying causal processes. After getting smoothing data we have applied normalization technique (min-max) upon that data.

In final phase we have implemented our proposed predictionLSTM with KNN model for forecasting of opening currency exchange rate on the basis of daily, weekly and monthly in separate way. Finally we have calculated prediction accuracy using performance measuring techniques such as MAPE and RMSE.

\section{TECHNOLOGY AND RESULT DEPICTION}

\section{A.Long Short-Term Memory (LSTM) Network}

Normal mechanism of RNN is processing the time sequences and also it is giving consent to a "memory" of earlier put in to preserve inner state of the network, whichever might be then utilised to persuade the network yield. In case of conventional RNN, it reveals a higher capability of modelling nonlinear time sequence dilemma, such as verbal communication recognition, speechmodelling, and picture captioning. On the other hand, conventional RNN is incapable to instruct the point in time sequence plus lengthy time lags. To defeatwith these types of problems a LSTM model is introduced.

LSTM model is generally treated as an extraordinary kind of RNN, which defeats the drawbacks and have designed to learn long term dependencies. LSTM networks are wellsuited for classifying, processing and making predictions based on time series data, since there can be lags of unknown duration between important events in a time series. This model consists of a bunch of building blocks of memory. In this case everybuilding blockencloses with solitary or more self-connected memory compartments and this can be represented through three gates, that is, input gate, forget gate, and output gate. Figure 2 shows the block diagram of long short-term memory [18-22].

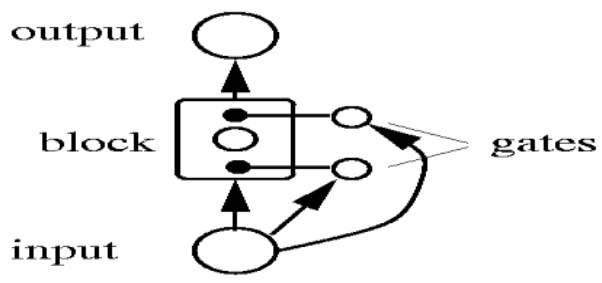

Figure 2: the building blockplot of LSTM

Assume the time series input as $Z=\left\{z_{1}, z_{2}, z_{3} \ldots, z_{T}\right\}$ and here $T$ is known as input sequence span. The numeral of inputs is named as $N$, all cells used in hidden layers are represented by $G$. $C$ is denoted as number of memory cells. The small alphabets such as $x, l$ plus $o$ is represented as input gate, forget gate with output gate correspondingly. Another symbol $p_{x y}$ is denoted as weight value connection rangefrom $x^{\text {th }}$ to $y^{\text {th }}$ part where as $b_{y}^{t}$ is known as system input fed to few unit of $y$ at time period $t$, and $a_{y}^{t}$, a value usedsubsequent to activation function retained in identical units. $r_{c}^{t}$ is treated as the compartmentstate on time period $t$. $\sigma$ is represented asgate activation function. Lastly $g$ plush both are known as input and output activation function. This algorithm is maintained by some equations which are as follows.

\section{Input Gates}

$$
\begin{aligned}
& b_{x}^{t}=\sum_{n=1}^{I} p_{n x} z_{n}^{t}+\sum_{h=1}^{G} p_{h x} a+\sum_{c=1}^{C} p_{c i} r_{c}^{t-1} \\
& a_{x}^{t}=\sigma\left(b_{x}^{t}\right)
\end{aligned}
$$

\section{Forget Gates}

$$
\begin{aligned}
& b_{l}^{t}=\sum_{n=1}^{I} p_{n l} i_{n}^{t}+\sum_{h=1}^{G} p_{h l} a_{h}^{t-1}+\sum_{c=1}^{C} p_{c l} r_{c}^{t-1} \\
& a_{l}^{t}=\sigma\left(b_{l}^{t}\right)
\end{aligned}
$$

Cells

$$
\begin{aligned}
& b_{c}^{t}=\sum_{n=1}^{I} p_{n c} i_{n}^{t}+\sum_{h=1}^{G} p_{h c} a_{h}^{t-1} \\
& r_{c}^{t}=a_{l}^{t} r_{c}^{t-1}+a_{x}^{t} g\left(b_{c}^{t}\right)
\end{aligned}
$$

\section{Output Gates}

$$
\begin{aligned}
& b_{o}^{t}=\sum_{n=1}^{I} I_{n o} i_{n}^{t}+\sum_{h=1}^{G} p_{h o} a_{h}^{t-1}+\sum_{c=1}^{C} p_{c o} r_{c}^{t-1} \\
& a_{o}^{t}=\sigma\left(a_{o}^{t}\right)
\end{aligned}
$$

\section{Cells Outputs}

$$
a_{c}^{t}=a_{o}^{t} h\left(r_{c}^{t}\right)
$$

\section{B. K-Nearest Neighbors Algorithm}

KNN algorithm is solitary of the most important classiertechniqueswhich is used for classification of data set and is also known as lazy algorithm. It is nonparametric innature. KNN algorithm is based on feature similarity: according with similarity amid the data points, classification might be possible. Basically, this algorithm is utilised for classification purpose. Most of cases researchers have implemented KNN method for prediction of financial products. The main motto of this algorithm is to classify the objects using majority voting procedure among neighbour data points and took the class which is getting major votes among all [23-26].

\section{Steps of KNN Algorithm}

Step 1: Before starting prediction procedure, first is to settle onconstraintabout parameter where $K$ is represented as number of nearest neighbours.

Step 2: In second step we compute the distance amongthe entire training samples plus query instances.

Step 3: After computation part is over, in next immediate step it sorts the distance whichdecides nearest neighbours on the basis of $k^{\text {th }}$ least amount distance.

Step 4: Next, it congregates the classr of the nearest neighbours.

Step 5: Lastly, it uses the simple majority voting procedure of the nearest neighbours at the same time as the prediction value of the query instance. 


\section{RESULT DISCUSSION}

In this paper we have chosen foreign currency exchange data for forecasting future rate. Data are scattered according with their country currencyexchange rate. For forecasting we have utilized hybrid LSTM and KNN model. In this manuscript we have used Intel i3 with 4GB Hard disk and windows 7 operating system for executing our experiment and complete code is written using python 3.

The proposed algorithm for experimental work follows the steps below:

Steps 1: Arrangement of Dataset:In this phase, we have collected the data set INR with JPY and CNY according to monthly, weekly and daily basis as depicted in section III.

Step 2: Smoothing Dataset: As raw data are noisy in nature. So we go for smoothing the raw data using technical indicators which help to eliminate outliers from a data set in order to make a pattern more visible [27-28]. We have different currency exchange data so we have applied technical indicators for individual data. The technical indicators used here are mentioned in (10), (11), (12) and (13).

$\operatorname{TSI}\left(b_{0}, r, s\right)=100 \times \frac{\operatorname{EMA}(\operatorname{EMA}(c, r), s)}{\operatorname{EMA}(\operatorname{EMA}(|c|, r), s)}$

$R S I=100-\frac{100}{1+\frac{E M A(W, c)}{E M A(G, c)}}$

$S M A_{k}=\frac{1}{2 P+1}(M A(k+P)+M A(k+P-1)+\cdots+$

$M A(k-P))$

$\% R=\frac{\text { high }_{\text {Pdays }}-\text { close }_{\text {Ptoday }}}{\text { high }_{\text {Pdays }}-\text { low }_{\text {Pdays }}} \times-100(13)$

Step 3: Scaling down the Dataset:Once smoothing is over we scale the data using min-max scaling. The main objective of scaling is to get a common scale by changing the numerical columns in currency exchange dataset without losing useful information. It also maintains the data integrity [28-29]. (14) shows the min-max normalization.

$\tilde{n}^{j k}=\frac{n_{j k}-n_{\min k}}{n_{\max k}-n_{\operatorname{mink}}}$

Where, $n_{j k}$ denotes $k^{\text {th }}$ attribute value of $j^{\text {th }}$ feature $n_{j}$, designed for the dataset, $k^{t h}$ minimum value is $n_{\min k}$ plus maximum value is $n_{\text {max }}$ in addition to normalized price of $j^{\text {th }}$ day is $\tilde{n}^{j}$.

Step 4: Effectuation of LSTM-K-NN method:Now our data are equipped for execution means it has an accurate format then after we have applied our forecasting model LSTM with KNN for predicting the original opening price of currency exchange data.LSTMs are recurrent networks where it replaces each neuron by a memory unit. The unit contains an actual neuron with a recurrent self-connection. The vector of outputs from all memory units is the output of the LSTM network. Hence, LSTM is used here mainly for classification of exchange price. The main aspire to use KNN is to classify a new sample point into a class out of several classes in the database.Also KNN is simple to interpret with low computation time and high predictability. So bothtechniques are working well in individual basis but when we hybrid these two they gave improved results in case of opening currency exchange price prediction. The result graphs are depicted in figure 3 and figure 4 for daily exchange price prediction of JPY/INR and CNY/INR respectively.

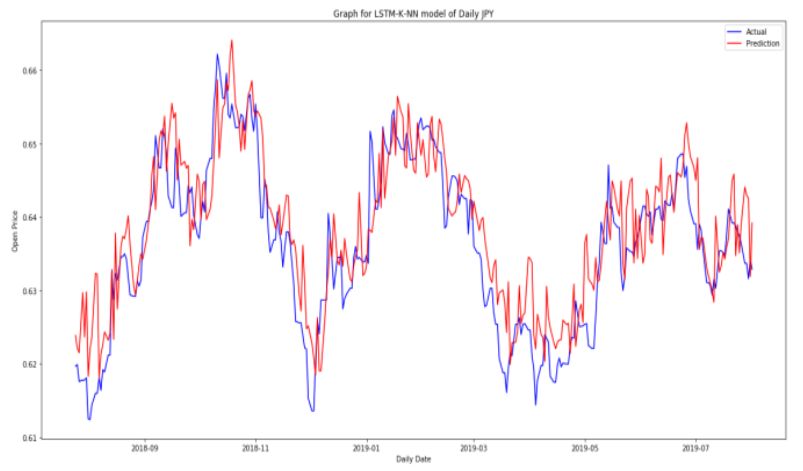

Figure 3:Graph of JPY/INRexchange rate prediction by LSTM-K-NN model on Daily basis

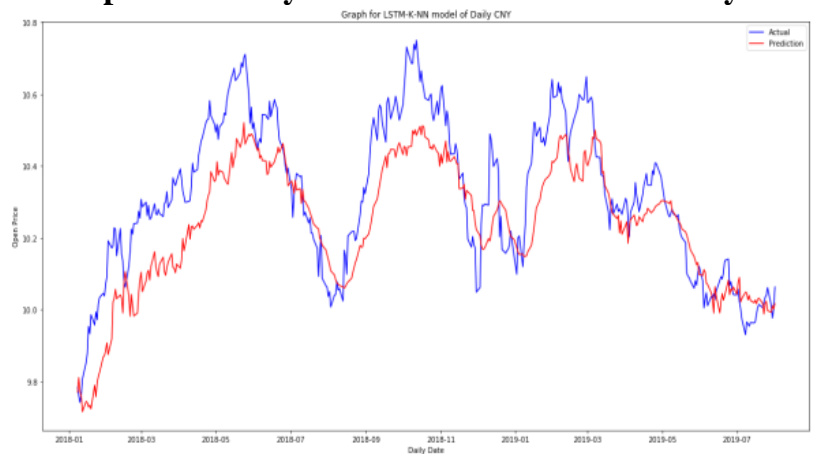

Figure 4: Graph of CNY/INR exchange rate prediction by LSTM-K-NN model on Daily basis

Step 5: Performance Observance:Toquantify the performance of our projectedforecasting model for predicting foreigncurrency exchange rate, we useroot mean squared error (RMSE) techniqueplus mean absolute percentage error (MAPE) which areshown below in (15) and (16).

$\mathrm{RMSE}=\sqrt{\frac{1}{D} \sum_{j=1}^{D}\left(a_{j}-\hat{a}_{i}\right)^{2}}$

$\mathrm{MAPE}=\frac{1}{D} \sum_{j=1}^{D}\left|\frac{a_{j}-\hat{a}_{j}}{a_{j}}\right| \times 100$

Where, $D$ is known as thesum of testing data, $a_{j}, \hat{a}_{j}$ are the required and forecasted outputs correspondingly.

The testing error values of daily, weekly and monthly predictionsmeasured by the techniquessuch as MAPE and RMSE are mentioned in table-II and table-III.

Published By:

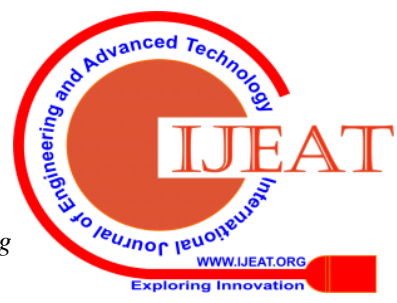


Table-II: JPY vs. INR testing error values by LSTM-K-NN Model

\begin{tabular}{|c|c|c|c|}
\hline & 1 Day & 1 Week & 1 Month \\
\hline MAPE & 0.0045 & 0.1561 & 0.0309 \\
\hline RMSE & 0.0057 & 0.0190 & 0.0376 \\
\hline
\end{tabular}

Table-III: CNY vs. INR testing error values by LSTM-K-NN Model

\begin{tabular}{|c|c|c|c|}
\hline & 1 Day & 1 Week & 1 Month \\
\hline MAPE & 0.1115 & 0.1697 & 0.3263 \\
\hline RMSE & 0.1352 & 0.2162 & 0.3919 \\
\hline
\end{tabular}

\section{CONCLUSION}

In this paper, we have presented the currency exchange opening price forecasting using a hybridLSTM-K-NN model. For this purpose our primary work was valid and accurate data collection. After getting accurate raw data we have applied technical indicators for smoothing,min-max normalization for scaling the dataset and finally we found standard dataset which was ready for execution. As LSTM handles gradient problem and allows learning of long term dependencies so it classified the currency exchange opening price well. KNN then classifies the profit or loss classes which exhibits its good predictability. Hence our proposed model gave better prediction results which were tested utilizing the standards such as MAPE and RMSE.

\section{REFERENCES}

1. Tenti, Paolo. "Forecasting foreign exchange rates using recurrent neural networks." Applied Artificial Intelligence 10.6 (1996): 567-582.

2. Yao, Jingtao, and Chew Lim Tan. "A case study on using neural networks to perform technical forecasting of forex." Neurocomputing 34.1-4 (2000): 79-98.

3. Chen, An-Sing, and Mark T. Leung. "Regression neural network for error correction in foreign exchange forecasting and trading." Computers \& Operations Research 31.7 (2004): 1049-1068.

4. Leigh, William, Ross Hightower, and Naval Modani. "Forecasting the New York stock exchange composite index with past price and interest rate on condition of volume spike." Expert Systems with Applications 28.1 (2005): 1-8.

5. Leu, Yungho, Chien-Pang Lee, and Yie-ZuJou. "A distance-based fuzzy time series model for exchange rates forecasting." Expert Systems with Applications 36.4 (2009): 8107-8114.

6. Pradhan, Rudra P., and Rajesh Kumar. "Forecasting exchange rate in India: An application of artificial neural network model." Journal of Mathematics Research 2.4 (2010): 111.

7. Pacelli, Vincenzo, VitoantonioBevilacqua, and Michele Azzollini. "An artificial neural network model to forecast exchange rates." Journal of Intelligent Learning Systems and Applications 3.02 (2011): 57.

8. Perwej, Yusuf, and AsifPerwej. "Forecasting of Indian Rupee (INR)/US Dollar (USD) currency exchange rate using artificial neural network." arXiv preprint arXiv:1205.2797 (2012).

9. Sermpinis, Georgios, Christian Dunis, Jason Laws, and Charalampos Stasinakis "Forecasting and trading the EUR/USD exchange rate with stochastic Neural Network combination and time-varying leverage." Decision Support Systems 54.1 (2012): 316-329.

10. Rehman, Mehreen, Gul Muhammad Khan, and Sahibzada Ali Mahmud. "Foreign currency exchange rates prediction using cgp and recurrent neural network." IERI Procedia 10 (2014): 239-244.

11. Das, Sauvik, Anisha Halder, Pavel Bhowmik, Aruna Chakraborty, Amit Konar, and A. K. Nagar. "Voice and facial expression based classification of emotion using linear support vector machine." In 2009 Second International Conference on Developments in eSystems Engineering IEEE (2009): 377-384.
12. Yao, Jingtao, and Chew Lim Tan. "A case study on using neural networks to perform technical forecasting of forex." Neurocomputing 34.1-4 (2000): 79-98.

13. Panda, Chakradhara, and V. Narasimhan. "Forecasting exchange rate better with artificial neural network." Journal of Policy Modeling 29.2 (2007): 227-236.

14. Yu, Lean, Kin Keung Lai, and Shouyang Wang. "Multistage RBF neural network ensemble learning for exchange rates forecasting." Neurocomputing 71.16-18 (2008): 3295-3302.

15. Anastasakis, Leonidas, and Neil Mort. "Exchange rate forecasting using a combined parametric and nonparametric self-organising modelling approach." Expert Systems with Applications 36.10 (2009): 12001-12011.

16. Gill, S. S., AmanjotKaur Gill, and Naveen Goel. "Indian currency exchange rate forecasting using neural networks." 2010 IEEE International Conference on Advanced Management Science (ICAMS 2010). 2010.

17. http://in.investing.com/currencies

18. Hochreiter, Sepp, and Jürgen Schmidhuber. "Long short-term memory." Neural computation 9.8 (1997): 1735-1780.

19. Ma, Xiaolei, Zhimin Tao, Yinhai Wang, Haiyang Yu, and Yunpeng Wang. "Long short-term memory neural network for traffic speed prediction using remote microwave sensor data." Transportation Research Part C: Emerging Technologies 54 (2015): 187-197.

20. Shao, Hongxin, and Boon-Hee Soong. "Traffic flow prediction with long short-term memory networks (LSTMs)." 2016 IEEE Region 10 Conference (TENCON).IEEE, 2016.

21. Zhao, Zheng, Weihai Chen, Xingming $\mathrm{Wu}$, Peter $\mathrm{CY}$ Chen, and Jingmeng Liu. "LSTM network: a deep learning approach for shortterm traffic forecast." IET Intelligent Transport Systems 11.2 (2017): 68-75.

22. Habtemichael, F., Mecit Cetin, and K. Anuar. "Methodology for quantifying incident-induced delays on freeways by grouping similar traffic patterns." Transportation Research Board 94th Annual Meeting. 2015.

23. Dell'Acqua, Pietro, Francesco Bellotti, Riccardo Berta, and Alessandro De Gloria. "Time-aware multivariate nearest neighbor regression methods for traffic flow prediction." IEEE Transactions on Intelligent Transportation Systems 16.6 (2015): 3393-3402.

24. Cai, Pinlong, Yunpeng Wang, Guangquan Lu, Peng Chen, Chuan Ding, and Jianping Sun. "A spatiotemporal correlative k-nearest neighbor model for short-term traffic multistep forecasting." Transportation Research Part C: Emerging Technologies 62 (2016): 21-34.

25. Sun, Bin, Wei Cheng, Prashant Goswami, and Guohua Bai. "Shortterm traffic forecasting using self-adjusting k-nearest neighbours." IET Intelligent Transport Systems 12.1 (2017): 41-48.

26. Bernaś, Marcin, Bartłomiej Płaczek, Piotr Porwik, and Teresa Pamuła. "Segmentation of vehicle detector data for improved k-nearest neighbours-based traffic flow prediction." IET intelligent transport systems 9.3 (2014): 264-274.

27. Nayak, Rudra Kalyan, Debahuti Mishra, and Amiya Kumar Rath. "A Naïve SVM-KNN based stock market trend reversal analysis for Indian benchmark indices." Applied Soft Computing 35 (2015): 670-680.

28. Nayak, Rudra Kalyan, Debahuti Mishra, and Amiya Kumar Rath. "An optimized SVM-k-NN currency exchange forecasting model for Indian currency market." Neural Computing and Applications 31.7 (2019): 2995-3021.

29. Nayak, Rudra Kalyan, Kuhoo, Debahuti Mishra, Amiya Kumar Rath and Ramamani Tripathy. "A Novel Look Back N feature approach towards prediction of crude oil price." Internationaljournal of engineering and technology, 3.34 (2018), 459-465. 


\section{AUTHORS PROFILE}

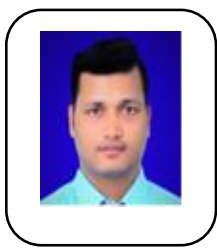

Dr.Rudra Kalyan Nayak, is an Associate Professor in CSE department at $\mathrm{K} \mathrm{L}$ (Deemed to be University), Andhra Pradesh, India. He holds his M.Tech in IT and Ph.D in CSEboth from S ' $\mathrm{O}$ ' A (Deemed to be) University, Odisha, India. He has $10+$ years of teaching, research and mentoring experience as a whole. He has interest in doing research in Financial Engineering, Computer Vision Bioinformatics and Machine Learning domain.

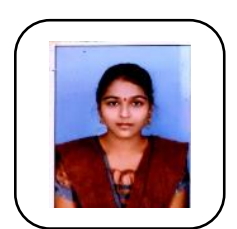

S.Y.H. Pavitra, is a final year student in CSE department at K L (Deemed to be University), Andhra Pradesh, India.

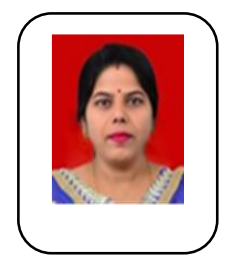

Dr. Ramamani Tripathy, works as an Assistant Professor in MCA department at USBM, Odisha, India. She has more than 10 years of academic, research and mentoring experience. She has got her Ph.D in CSE from S'O'A University, Odisha. She likes to do research in Data mining, Bioinformatics and Financial Engineering domain.

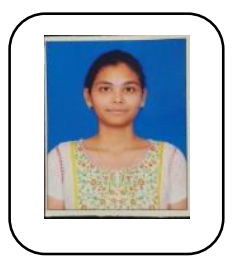

K. Prathyusha, is a final year student in CSE department at K L (Deemed to be University), Andhra Pradesh, India. 